\title{
Performance of an Off-line Signature Verification Method based on Texture Features on a Large Indic-script Signature Dataset
}

\author{
Srikanta Pal \\ School of ICT, Griffith \\ University, Australia \\ srikanta.pal@griffithuni.edu.au
}

\author{
Alireza Alaei \\ School of ICT, Griffith \\ University, Australia \\ alireza20alaei@yahoo.com
}

\author{
Umapada Pal \\ Indian Statistical Institute \\ Kolkata, India \\ umapada@isical.ac.in
}

\author{
Michael Blumenstein \\ School of ICT, Griffith \\ University, Australia \\ m.blumenstein@griffith.edu.au
}

\begin{abstract}
In this paper, a signature verification method based on texture features involving off-line signatures written in two different Indian scripts is proposed. Both Local Binary Patterns (LBP) and Uniform Local Binary Patterns (ULBP), as powerful texture feature extraction techniques, are used for characterizing off-line signatures. The Nearest Neighbour (NN) technique is considered as the similarity metric for signature verification in the proposed method. To evaluate the proposed verification approach, a large Bangla and Hindi off-line signature dataset (BHSig260) comprising $6240 \quad(260 \times 24)$ genuine signatures and $7800(260 \times 30)$ skilled forgeries was introduced and further used for experimentation. We further used the GPDS-100 signature dataset for the comparison. The experiments were conducted, and the verification accuracies were separately computed for the LBP and ULBP texture features. There were no remarkable changes in the results obtained applying the LBP and ULBP features for verification when the BHSig260 and GPDS-100 signature datasets used for experimentation.
\end{abstract}

Keywords: Off-line signature verification; Off-line signature dataset; Local Binary Pattern (LBP); Texture features.

\section{INTRODUCTION}

Biometrics play an increasingly important role in different applications of person/human identification and authentication. In the area of biometrics, various technologies have been developed, such as those based on fingerprints, iris, face, voice, handwritten signatures, and hands, to handle the problem of person identification and authentication.

Handwritten signatures occupy a very special place in this wide set of biometric traits and many automatic signature recognition/verification systems have also been developed in the literature [3]. Signature identification method decides the signature group among the number of groups that the claimed signature belongs to. On the other hand, signature verification method confirms or rejects a person's claimed signature.

Signature verification systems, and the associated techniques used to solve the inherent problem of authentication, can be divided into two categories: i) on-line, and ii) off-line. In an on-line method [1], sequential data, such as handwriting speed and pen pressure, is used for verification and identification purposes. In an off-line method [2], signature written on paper is scanned to be used for the verification process. Handwriting signature recognition in off-line mode is more difficult than in on-line systems as a lot of dynamic information is lost during the scanning process. Hence, on-line signature verification methods have generally achieved higher accuracies compared to the off-line methods. Nevertheless, off-line systems have a significant advantage in that they do not require access to special processing devices when the signatures are produced [3].

In the literature of off-line signature verification many research works based on different features, such as texture features and in particular local binary patterns (LBP)-based texture feature, were proposed [2-9]. A few research works, which employ signatures of Indian scripts, were also introduced in the field of non-English based signature verification [10-15].

A method based on local directional patterns (LDP) for off-line handwritten signature verification was presented in [4]. In this paper [4], the usefulness of texture-based measures was explored by the means of local binary patterns (LBP) and local directional pattern (LDP) features on the binarized signatures. The experiments were conducted on the MCYT75, GPDS300 and GPDS960 signature corpuses and the results showed that the LDP features were suitable for automatic verification of black and white static signatures. The results were obtained by training a Support Vector Machine (SVM) classifier with genuine samples and random forgeries, while random and skilled forgeries were used for testing purposes. In a study, Serdouk et al. [5] proposed a combination of two feature sets, employing a new LBPbased feature that is called Orthogonal Combination of Local Binary Patterns (OC-LBP). In addition, they proposed a topological feature (Longest Run Feature) that considers the longest pixel sequence within the signature image. The features were applied to SVM classifier to compute the outcome of the verification system. A signature verification method using the LBP was presented in [6]. The signature models were trained with genuine signatures on white backgrounds, and tested with other genuine and forged signatures mixed with different backgrounds. Results showed that a basic version of the LBP or local derivative and directional patterns were more robust than rotation invariant uniform LBP or Gray-Level Co-Occurrence Matrix (GLCM) features using an SVM with histogram-oriented kernels as a classifier for signature verification [6]. Another 
off-line signature verification system based on local histogram features was presented in [7]. In the method presented in [7], a signature was initially divided into zones using both the Cartesian and polar coordinate systems. The histogram of oriented gradients (HOG) and the histogram of local binary patterns (LBP) were calculated as features for each zone. The classification was performed using SVMs, where two different approaches for training, namely global and user-dependent SVMs, were investigated. The fusion of all classifiers (global and user-dependent classifiers) achieved a $15.41 \%$ equal error rate with skilled forgery tests considering the GPDS-160 signature dataset.

From the literature surveyed, it can be noted that impressive progress was achieved in the field of signature verification using various texture features when considering signatures written in Latin-based scripts for the experiments. However, the methods presented in the SigWiComp2013 indicate that the problem of signature identification/ verification still remains a challenging problem [9], when the number of signatures' classes is increasing. Texture-based features considering non-Latin based signatures have not been used for verification purposes. In this research work, therefore, a signature verification method based on texture features is presented to analyse verification performance on a large-scale off-line Bangla and Hindi signature dataset. Two different types of texture features called local binary patterns (LBP) and uniform local binary patterns (ULBP) are extracted from each signature, and a nearest neighbour classifier using the Euclidian distance is considered for verification. The large-scale off-line Bangla and Hindi signature dataset used for the experimentation called as BHSig260 dataset contains $6240 \quad(260 \times 24)$ genuine signatures and $7800(260 \times 30)$ skilled forgeries. The dataset is further introduced to the research community and made publicly available for research purposes.

The remainder of this paper is structured as follows. The dataset details are presented in Section II. Section III represents the proposed technique. The experimental results and comparison of performance are presented in Section IV. Finally, conclusions are drawn in Section V.

\section{BENGALI AND HiNDI SIGNATURE DATASET DETAILS}

In the field of signature verification, there is a sparseness of publicly available signature datasets. The quality of the available datasets also varies, as there has been no standard data collection protocol for creation of datasets. Besides, it is very costly to create a large corpus with different types of forgeries, especially skilled forgeries. Two off-line signature databases, which are widely used in the literature, are GPDS960 [19] and MCYT [20]. As there has been no public signature corpus available for Bangla and Hindi script, it was necessary to create a dataset of off-line Bangla and Hindi signatures. This Bangla and Hindi signature (BHSig260) dataset consists of 260 sets of handwritten off-line signatures of which 100 sets were written in Bangla script for the Bangla part and the rest (160 sets) were written in Hindi script for the Hindi part of the BHSig260 dataset. The handwritten off-line signatures were collected from 260 different individuals with different educational backgrounds and ages. Each set consists of 24 genuine signatures and 30 skilled forgeries. Signatures were collected during 2 different sessions. In the first session, the genuine signatures were collected, whereas in the second session the skilled forgeries were collected, showing a genuine signature to an individual to train and mimic the forgeries. A total number of 6240 genuine and 7800 skilled forgery signatures were collected from all 260 individuals. The collected data were acquired using a Flatbed scanner with the resolution of 300DPI in grey scale and stored in TIFF format (Tagged Image File Format). A histogram-based threshold technique was applied for binarization to convert digitized grey-level images to two-tone images. The skilled forgery signatures collected are quite similar to the genuine signatures, which makes the dataset quite a challenging one. To have an idea about the complexity of the forged signatures, some binary genuine signature samples of the BHSig260 dataset with their corresponding forgeries are displayed in Table I. The BHSig260 dataset introduced in this research work is publicly available for research purposes.

TABLE I. GENUINE AND FORGED SIGNATURE SAMPLES OF THREE DIFFERENT CLASSES FORM EACH PART OF THE BHSIG260 DATASET.

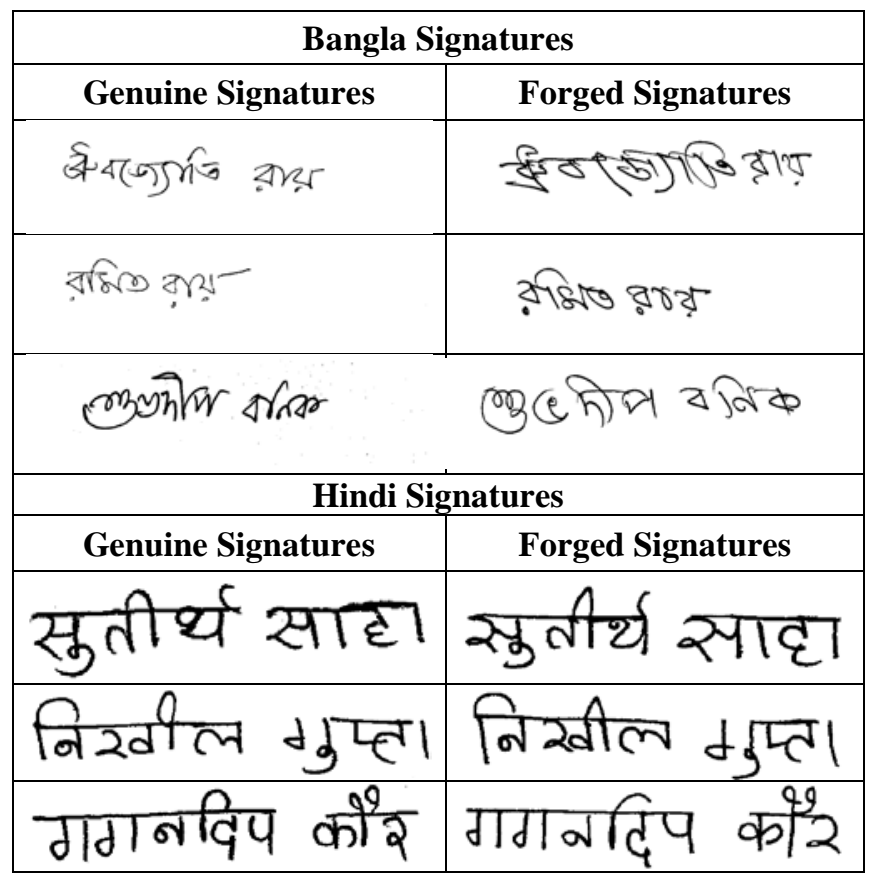

\section{PROPOSED METHOD}

To have a quick overview of our proposed signature verification method and its steps, the block diagram of the proposed method is presented in Fig. 1. The proposed method similar to most of the methods in the literature is composed of pre-processing, feature extraction, and verification steps. Details of each step are described in the following subsections.

\section{A. Preprocessing}

In the pre-processing step, a mean filter is employed on the signature images to remove any noise present. The 
minimum bounding box of the signature images are also determined to have stable feature sets.

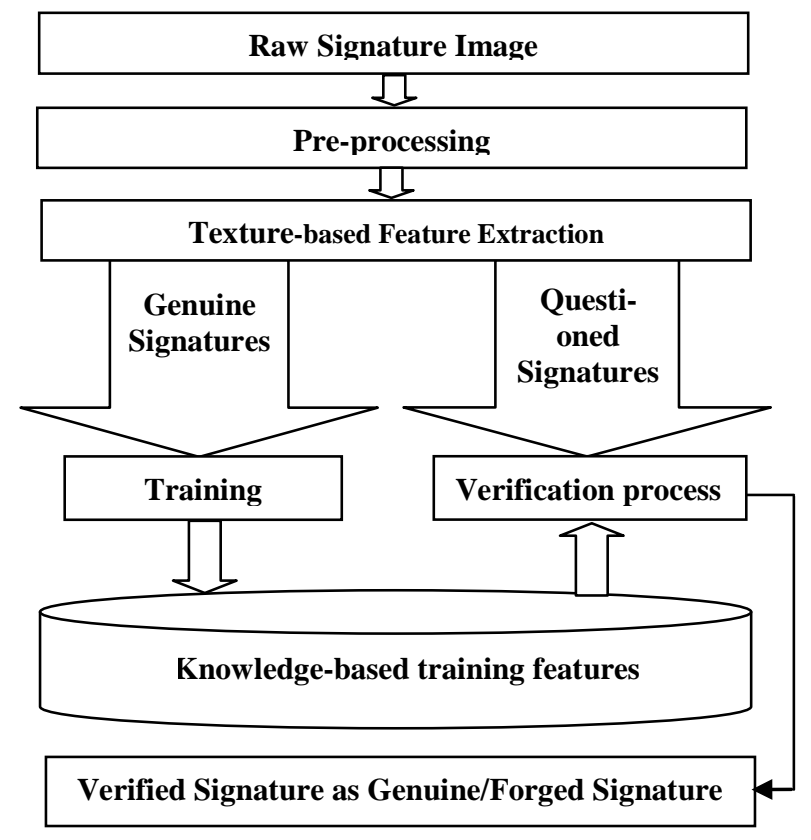

Fig. 1. Overview of the proposed signature verificaion method.

\section{B. Feature Extraction Method}

Feature extraction is a vital step in any pattern recognition system. Different methods have been proposed in the off-line signature verification literature to perform the extraction of features from signature images [16]. The texture features and in particular LBP and ULBP features have shown their strength on various signatures types having different patterns in the literature [3-7]. In this research work the LBP-based feature extraction techniques $[17,18]$ used in the literature for signature verification [4-7] are considered for feature extraction purposes. Since, most of the LBP patterns of an image are uniform patterns, ULBP are further computed as a lower dimension feature set compared to the LBP. This is the only difference between the LBP and ULBP features. Furthermore, the LBP and ULBP features are chosen for signature verification in this work to study the behaviour of those features on Bangla and Hindi signatures, as such kinds of signatures are mostly textual patterns. In the following, brief descriptions of two feature sets used in this paper are discussed.

Local binary pattern (LBP): Local binary pattern (LBP) is a powerful feature proposed to capture the texture in objects [17]. In the basic LBP method, a grey scale image is processed in such a way that a binary code is generated for each pixel in the image. This code encodes whether the intensities of the neighbouring pixels are greater or less than the current pixel's intensity. So, for instance in a $3 \times 3$ neighbourhood with the current pixel being the centre, a binary code of length 8 is generated consisting of 0 s and $1 \mathrm{~s}$, according to the relative intensities of the neighbours. A histogram is then computed to count the number of occurrences of each binary code, describing the proportion of common textural patterns in an image $[17,18]$. Applying the LBP method for feature extraction, a feature set of size 256 is obtained from a signature image used further for signature verification.

Uniform Local Binary Pattern (ULBP): A local binary pattern is called uniform if the binary pattern contains at most two bitwise transitions from 0 to 1 or vice versa when the bit pattern is considered circular. For example, the patterns 00000000 (0 transitions), 01110000 (2 transitions) and 11001111 (2 transitions) are uniform whereas the patterns 11001001 (4 transitions) and 01010011 (6 transitions) are not.

In the computation of the LBP histogram, uniform patterns are used so that the histogram has a separate bin for every uniform pattern and all non-uniform patterns are assigned to a single bin [18]. Employing the ULBP feature extraction method on a signature image, a feature set of size 59 is computed used for the signature verification in this research work.

\section{Verification Technique}

A Nearest Neighbour (NN) classifier with a Euclidian distance measure achieves consistently good performance without a priori assumptions about the distributions of the training samples, amongst the various methods of supervised statistical pattern recognition. In this paper, the NN is used for signature verification. Following the pre-processing and feature extraction methods applied on a test signature, Euclidian distances between the test features and the corresponding training features are computed. The minimum distance is obtained and compared to an acceptance/rejection threshold value obtained for that particular class. The threshold value is adaptively computed for each signature class considering the statistical mean and standard deviation of all distance values obtained between training features of that class. Since, we considered 8 and 12 genuine signatures from each class for training, $28(8 \times 7 / 2)$ and $66(12 \times 11 / 2)$ distances were computed for each class, respectively. The statistical mean $\left(\mathrm{Mean}_{\mathrm{c}}\right)$ and standard deviation $\left(\mathrm{STD}_{\mathrm{c}}\right)$ values of the computed distances were computed for each class $(c)$. Different thresholds $\left(\Theta_{c}\right)$ were obtained considering a linear combination of $\operatorname{Mean}_{\mathrm{c}}$ and $\operatorname{STD}_{\mathrm{c}}\left(\Theta_{\mathrm{c}}=\operatorname{Mean}_{\mathrm{c}}+\right.$ $K \times \mathrm{STD}_{\text {c }}$ ) for each class to be used as acceptance/rejection criteria at the verification stage.

\section{RESULTS AND DISCUSSION}

The BHSig260 as a large dataset of Bangla and Hindi signatures was initially used for experiments in this research work. For computing the experimental results, the first 8 and 12 genuine signatures from each individual (signature class) were considered for training during different experiments. No forged signature samples were used in the training phase. The remaining 16 and 12 genuine signatures and 30 skilled forgeries from each individual in the signature dataset were considered for testing purposes, accordingly. The threshold values were determined using the mean and standard deviation values when considering different values for $K$. We 
further obtained the results using only Bangla and Hindi parts of the BHSig260 dataset separately to observe the performance of the different feature sets on signatures written in different scripts (Bangla and Hindi). The accuracies were computed based on different threshold values for the LBP and ULBP features extracted from the test images. This is executed to observe how each feature type performs separately for different threshold values. The GPDS-100 signature dataset is also considered for the comparison of the results on a benchmark. It contains 24 genuine signatures and 24 forgeries written by 100 individuals. In total, $2400(100 \times 24)$ genuine signatures and the same number of forgeries (2400) are available in the GPDS-100 signature dataset. The signatures were scanned in grey-scale with 600 dpi resolution and they were stored in PNG format [19].

The outcome of the system was measured in terms of False Acceptance Rate (FAR), False Rejection Rate (FRR) and Average Error Rate (AER). We also obtained the results where FRRs are equal to FARs to report Equal Error Rates (ERR). The accuracies obtained in these experiments are described and shown in tabular form in the following subsections.

\section{A. Experiment using LBP:}

In this step of experimentation, different results obtained considering the LBP features and different numbers of signatures for training are shown in Table II. Using different threshold values in the proposed signature verification method, the results were obtained to demonstrate the behaviour of FAR, FRR and AER values on the BHSig260 dataset. The ERR was also obtained when FAR and FRR are equal. From Table II, it is observed that the EER was obtained in the experiments when $K$ was considered to be 0.64 using 12 signatures from each class for training. The graphical representation of the FAR and AER results (Table II) obtained considering different acceptance/rejection thresholds $\left(\Theta_{\text {c) }}\right.$ in relation to different values of $K$ when 12 signatures were used for training is shown in Fig. 2.

TABLE II. EXPERIMENTAL ACCURACIES ON THE BHSIG260 DATASET CONSIDERING LBP-BASED FEATURES USING VARIOUS THRESHOLDS AND DIFFERENT SIGNATURES FROM EACH CLASS FOR TRAINING

\begin{tabular}{|c|c|c|c|c|c|c|c|}
\hline \multirow{2}{*}{$\begin{array}{c}K \\
\text { Value }\end{array}$} & \multicolumn{3}{|c|}{$\begin{array}{l}8 \text { signatures from each } \\
\text { class for training }\end{array}$} & \multirow{2}{*}{$\begin{array}{c}K \\
\text { Value }\end{array}$} & \multicolumn{3}{|c|}{$\begin{array}{l}12 \text { signatures from each } \\
\text { class for training }\end{array}$} \\
\hline & $\begin{array}{c}\text { FAR } \\
(\%)\end{array}$ & $\begin{array}{c}\text { FRR } \\
(\%)\end{array}$ & $\begin{array}{c}\text { AER } \\
(\%)\end{array}$ & & $\begin{array}{c}\text { FAR } \\
(\%)\end{array}$ & $\begin{array}{c}\text { FRR } \\
(\%)\end{array}$ & $\begin{array}{c}\text { AER } \\
(\%)\end{array}$ \\
\hline-0.5 & 43.32 & 26.47 & 34.89 & -0.5 & 47.91 & 23.59 & 35.75 \\
\hline 0 & 38.12 & 29.33 & 33.72 & 0 & 42.10 & 26.22 & 34.16 \\
\hline 0.4 & 33.26 & 32.79 & 33.02 & 0.5 & 34.71 & 30.42 & 32.56 \\
\hline 0.42 & 33.06 & 33.06 & 33.06 & 0.6 & 33.26 & 31.86 & 32.56 \\
\hline 0.5 & 31.67 & 34.13 & 32.90 & 0.64 & 32.72 & 32.72 & 32.72 \\
\hline 0.7 & 28.58 & 37.07 & 32.82 & 0.7 & 31.60 & 34.23 & 32.92 \\
\hline 1 & 22.95 & 46.56 & 34.76 & 1 & 24.40 & 43.69 & 34.04 \\
\hline 1.5 & 1.94 & 93.37 & 47.65 & 1.2 & 16.44 & 57.98 & 37.21 \\
\hline
\end{tabular}

The results obtained employing the LBP-based features in the experiments with different threshold values and different numbers of signatures for training on the Bangla and Hindi parts of the BHSig260 dataset are further shown in
Table III and Table IV, respectively. From Table III, it is observed that the best performance with $33.70 \%$ AER was obtained on the Bangla part of the BHSig260 in the experiments when $K$ was considered to be 0.9. The results obtained in Table IV were based on the Hindi part of the BHSig260 dataset using different acceptance/rejection thresholds. The results presented in Table IV indicate that $77.80 \%(100 \%-22.20 \%)$ of the Hindi signatures were correctly verified using the LBP features.

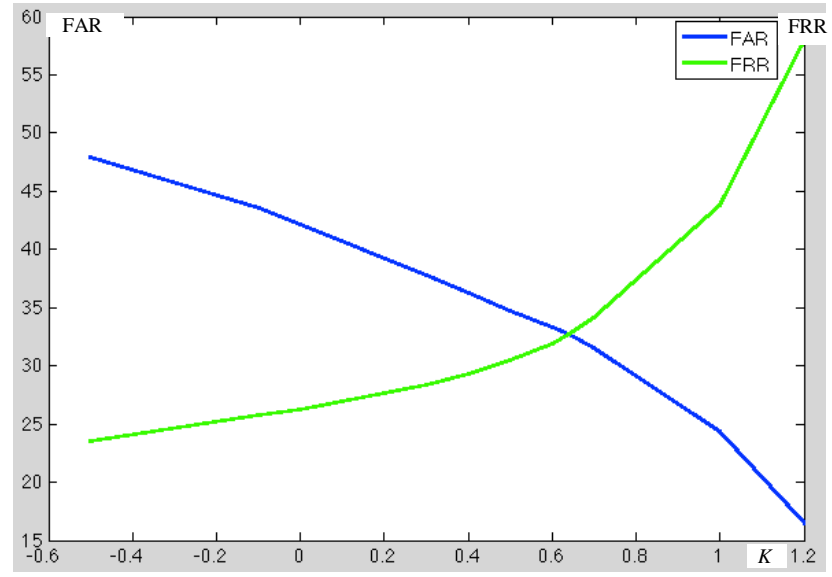

Fig.2. The graphical representation of the FAR and AER results (Table II) obtained considering different acceptance/rejection thresholds $\left(\Theta_{c}\right)$ in relation to the values of $K$ when 12 signatures were used for training.

TABLE III. EXPERIMENTAL ACCURACIES ON THE BANGLA PART OF THE BHSIG260 DATASET CONSIDERING LBP-BASED FEATURES USING VARIOUS THRESHOLDS AND DIFFERENT SIGNATURES FROM EACH CLASS FOR TRAINING

\begin{tabular}{|c|c|c|c|c|c|c|c|}
\hline \multirow{2}{*}{$\begin{array}{c}K \\
\text { Value }\end{array}$} & \multicolumn{3}{|c|}{$\begin{array}{l}8 \text { signatures from each } \\
\text { class for training }\end{array}$} & \multirow{2}{*}{$\begin{array}{c}K \\
\text { Value }\end{array}$} & \multicolumn{3}{|c|}{$\begin{array}{l}12 \text { signatures from each } \\
\text { class for training }\end{array}$} \\
\hline & $\begin{array}{c}\text { FAR } \\
(\%)\end{array}$ & $\begin{array}{c}\text { FRR } \\
(\%)\end{array}$ & $\begin{array}{c}\text { AER } \\
(\%)\end{array}$ & & $\begin{array}{l}\text { FAR } \\
(\%)\end{array}$ & $\begin{array}{c}\text { FRR } \\
(\%)\end{array}$ & $\begin{array}{c}\text { AER } \\
(\%)\end{array}$ \\
\hline-0.5 & 70.13 & 5.44 & 37.79 & -0.5 & 75.60 & 3.25 & 39.43 \\
\hline 0 & 65.20 & 7.75 & 36.48 & 0.5 & 62.03 & 6.58 & 34.31 \\
\hline 0.5 & 56.57 & 12.63 & 34.60 & 0.9 & 53.73 & 13.67 & 33.70 \\
\hline 0.9 & 46.40 & 22.94 & 34.67 & 1.1 & 43.17 & 24.42 & 33.79 \\
\hline 1.1 & 35.35 & 35.35 & 35.35 & 1.196 & 34.12 & 34.12 & 34.12 \\
\hline 1.2 & 27.40 & 45.13 & 36.26 & 1.2 & 33.50 & 34.75 & 34.13 \\
\hline 1.3 & 20.07 & 56.56 & 38.31 & 1.3 & 22.20 & 52.25 & 37.23 \\
\hline 1.5 & 3.50 & 86.56 & 45.03 & 1.5 & 0.63 & 89.42 & 45.03 \\
\hline
\end{tabular}

TABLE IV. EXPERIMENTAL ACCURACIES ON THE HINDI PART OF THE BHSIG260 DATASET CONSIDERING LBP-BASED FEATURES USING VARIOUS THRESHOLDS AND DIFFERENT SIGNATURES FROM EACH CLASS FOR TRAINING

\begin{tabular}{|c|c|c|c|c|c|c|c|}
\hline \multirow{2}{*}{$\begin{array}{c}K \\
\text { Value }\end{array}$} & \multicolumn{3}{|c|}{$\begin{array}{l}8 \text { signatures from each } \\
\text { class for training }\end{array}$} & \multirow{2}{*}{$\begin{array}{c}K \\
\text { Value }\end{array}$} & \multicolumn{3}{|c|}{$\begin{array}{l}12 \text { signatures from each } \\
\text { class for training }\end{array}$} \\
\hline & $\begin{array}{c}\text { FAR } \\
(\%)\end{array}$ & $\begin{array}{c}\text { FRR } \\
(\%)\end{array}$ & $\begin{array}{c}\text { AER } \\
(\%)\end{array}$ & & $\begin{array}{c}\text { FAR } \\
(\%)\end{array}$ & $\begin{array}{c}\text { FRR } \\
(\%)\end{array}$ & $\begin{array}{c}\text { AER } \\
(\%)\end{array}$ \\
\hline-1.5 & 2.42 & 90.20 & 46.31 & -1.5 & 0.40 & 97.86 & 49.13 \\
\hline-1.1 & 20.83 & 34.41 & 27.62 & -1.1 & 20.88 & 31.25 & 26.06 \\
\hline-1 & 23.42 & 28.09 & 25.75 & -1 & 24.47 & 24.47 & 24.47 \\
\hline-0.935 & 25.07 & 25.07 & 25.07 & -0.9 & 27.67 & 19.32 & 23.49 \\
\hline-0.9 & 25.83 & 23.55 & 24.69 & -0.7 & 32.63 & 12.71 & 22.67 \\
\hline-0.5 & 32.33 & 13.13 & 22.73 & -0.5 & 36.17 & 8.23 & 22.20 \\
\hline 0 & 39.02 & 8.16 & 23.59 & 0 & 44.33 & 4.48 & 24.41 \\
\hline 1 & 49.48 & 4.41 & 26.95 & 1 & 56.35 & 1.77 & 29.06 \\
\hline
\end{tabular}




\section{B. Experiments using ULBP:}

In this step of experimentation, different results obtained considering the ULBP features and different numbers of signatures for training when considering the BHSig260 dataset for experiment are shown in Table V. Employing the proposed signature verification method using different threshold values on the BHSig260 dataset, the results were obtained to demonstrate the behaviour of the proposed signature verification method using the ULBP features. From Table $\mathrm{V}$ it is observed that the lowest AER was obtained in the experiment when the threshold value was set to be 0.6 and 12 signatures from each class were used for training.

The results obtained employing ULBP-based features in the experiments with different threshold values and different numbers of signatures for training on the Bangla and Hindi parts of the BHSig260 dataset are further shown in Table VI and Table VII, respectively. From Table VI and VII, it is evident that the ERR results were obtained for the Bangla and Hindi signature parts of the BHSig260 dataset when 12 signatures considered for the training and the values of $K$ were set to 1.194 and -1 , respectively. From Table V, VI and VII, it is also noted that training the proposed method using 12 signatures from each signature class provided slightly better signature verification results compared to the results obtained considering 8 signatures from each class for training.

TABLE V. EXPERIMENTAL ACCURACIES ON THE BHSIG260 DATASET CONSIDERING ULBP-BASED FEATURES USING VARIOUS THRESHOLDS AND DIFFERENT SIGNATURES FROM EACH CLASS FOR TRAINING

\begin{tabular}{|c|c|c|c|c|c|c|c|}
\hline \multirow{2}{*}{$\begin{array}{c}\boldsymbol{K} \\
\text { Value }\end{array}$} & \multicolumn{2}{|c|}{$\begin{array}{c}\text { 8 signatures from each } \\
\text { class for training }\end{array}$} & \multirow{2}{*}{$\boldsymbol{K}$} & \multicolumn{3}{|c|}{$\begin{array}{c}\text { 12 signatures from each } \\
\text { class for training }\end{array}$} \\
\cline { 2 - 4 } \cline { 7 - 9 } & $\begin{array}{c}\text { FAR } \\
\text { Value }\end{array}$ & $\begin{array}{c}\text { FRR } \\
\mathbf{( \% )}\end{array}$ & $\begin{array}{c}\text { AER } \\
\mathbf{( \% )}\end{array}$ & & $\begin{array}{c}\text { FAR } \\
\mathbf{( \% )}\end{array}$ & $\begin{array}{c}\text { FRR } \\
\mathbf{( \% )}\end{array}$ & $\begin{array}{c}\text { AER } \\
\mathbf{( \% )}\end{array}$ \\
\hline-0.5 & 43.32 & 26.47 & 34.89 & -0.5 & 47.91 & 23.59 & 35.75 \\
\hline 0 & 38.12 & 29.33 & 33.72 & 0 & 42.10 & 26.22 & 34.16 \\
\hline 0.4 & 33.24 & 32.79 & 33.02 & 0.5 & 34.71 & 30.51 & 32.61 \\
\hline 0.42 & $\mathbf{3 3 . 0 5}$ & $\mathbf{3 3 . 0 5}$ & $\mathbf{3 3 . 0 5}$ & 0.6 & 33.26 & 31.86 & 32.56 \\
\hline 0.5 & 31.67 & 34.13 & 32.90 & $\mathbf{0 . 6 4}$ & $\mathbf{3 2 . 7 2}$ & $\mathbf{3 2 . 7 2}$ & $\mathbf{3 2 . 7 2}$ \\
\hline 0.7 & 28.55 & 37.07 & 32.81 & 0.7 & 31.58 & 34.20 & 32.89 \\
\hline 1 & 22.96 & 46.56 & 34.76 & 1 & 24.37 & 43.75 & 34.06 \\
\hline 1.5 & 1.85 & 93.34 & 47.59 & 1.2 & 16.42 & 58.04 & 37.23 \\
\hline
\end{tabular}

TABLE VI. EXPERIMENTAL ACCURACIES ON THE BANGLA PART OF THE BHSIG260 DATASET CONSIDERING ULBP-BASED FEATURES USING DIFFERENT SIGNATURES FROM EACH CLASS FOR TRAINING

\begin{tabular}{|c|c|c|c|c|c|c|c|}
\hline \multirow{2}{*}{$\begin{array}{c}K \\
\text { Value }\end{array}$} & \multicolumn{3}{|c|}{$\begin{array}{l}8 \text { signatures from each } \\
\text { class for training }\end{array}$} & \multirow{2}{*}{$\begin{array}{c}K \\
\text { Value }\end{array}$} & \multicolumn{3}{|c|}{$\begin{array}{l}12 \text { signatures from each } \\
\text { class for training }\end{array}$} \\
\hline & $\begin{array}{c}\text { FAR } \\
(\%)\end{array}$ & $\begin{array}{l}\text { FRR } \\
(\%)\end{array}$ & $\begin{array}{c}\text { AER } \\
(\%)\end{array}$ & & $\begin{array}{c}\text { FAR } \\
(\%)\end{array}$ & $\begin{array}{c}\text { FRR } \\
(\%)\end{array}$ & $\begin{array}{c}\text { AER } \\
(\%)\end{array}$ \\
\hline-0.5 & 70.13 & 5.44 & 37.79 & -0.5 & 75.53 & 3.25 & 39.39 \\
\hline 0 & 65.13 & 7.75 & 36.44 & 0 & 69.70 & 4.42 & 37.06 \\
\hline 0.5 & 56.57 & 12.63 & 34.60 & 0.5 & 62.00 & 6.58 & 34.29 \\
\hline 1 & 41.17 & 28.06 & 34.61 & 0.9 & 53.63 & 13.67 & 33.65 \\
\hline 1.095 & 35.13 & 35.13 & 35.13 & 1 & 50.13 & 18.25 & 34.19 \\
\hline 1.2 & 27.17 & 45.19 & 36.18 & 1.194 & 33.82 & 33.82 & 33.82 \\
\hline 1.3 & 19.80 & 56.63 & 38.21 & 1.2 & 33.20 & 34.75 & 33.98 \\
\hline 1.5 & 3.40 & 86.75 & 45.08 & 1.5 & 0.60 & 89.50 & 45.05 \\
\hline
\end{tabular}

TABLE VII. EXPERIMENTAL ACCURACIES ON THE HINDI PART OF THE BHSIG260 DATASET CONSIDERING ULBP-BASED FEATURES USING VARIOUS THRESHOLDS AND DIFFERENT SIGNATURES FROM EACH CLASS FOR TRAINING

\begin{tabular}{|c|c|c|c|c|c|c|c|}
\hline \multirow{2}{*}{$\begin{array}{c}K \\
\text { Value }\end{array}$} & \multicolumn{3}{|c|}{$\begin{array}{l}8 \text { signatures from each } \\
\text { class for training }\end{array}$} & \multirow{2}{*}{$\begin{array}{c}K \\
\text { Value }\end{array}$} & \multicolumn{3}{|c|}{$\begin{array}{l}12 \text { signatures from each } \\
\text { class for training }\end{array}$} \\
\hline & $\begin{array}{c}\text { FAR } \\
(\%)\end{array}$ & $\begin{array}{c}\text { FRR } \\
(\%)\end{array}$ & $\begin{array}{c}\text { AER } \\
(\%)\end{array}$ & & $\begin{array}{c}\text { FAR } \\
(\%)\end{array}$ & $\begin{array}{c}\text { FRR } \\
(\%)\end{array}$ & $\begin{array}{c}\text { AER } \\
(\%)\end{array}$ \\
\hline-1.5 & 2.40 & 90.16 & 46.28 & -1.5 & 0.44 & 97.86 & 49.15 \\
\hline-1 & 23.44 & 28.09 & 25.76 & -1.1 & 20.92 & 31.25 & 26.08 \\
\hline-0.9 & 24.69 & 24.69 & 24.69 & -1 & 24.47 & 24.47 & 24.47 \\
\hline-0.7 & 29.06 & 16.13 & 22.60 & -0.9 & 27.67 & 19.32 & 23.49 \\
\hline-0.5 & 32.33 & 13.13 & 22.73 & -0.5 & 36.17 & 8.23 & 22.20 \\
\hline 0 & 39.02 & 8.16 & 23.59 & 0 & 44.33 & 4.48 & 24.41 \\
\hline 0.5 & 44.58 & 6.17 & 25.38 & 0.5 & 51.10 & 2.66 & 26.88 \\
\hline 1 & 49.48 & 4.41 & 26.95 & 1 & 56.35 & 1.77 & 29.06 \\
\hline
\end{tabular}

\section{Comparison of performance}

The ERR results obtained considering the LBP and ULBP features in the proposed signature verification methods when 12 genuine signatures from each class were used for training are demonstrated in Table VIII. From Table VIII, it is clear that $66.18 \%, 75.53 \%$, and $87.28 \%$ of the testing Bangla, Hindi and the BHSig260 signatures were verified correctly. Moreover, the LBP and ULBP features were almost equally performed in the verification of Bangla and Hindi signatures.

TABLE VIII. COMPARISON OF THE RESULTS OBTAINED USING DIFFERENT FEATURES CONSIDERING THE BHSIG260 SIGNATURE DATASET, THE BANGLA AND HINDI PARTS OF THE BHSIG260 .

\begin{tabular}{|l|c|c|c|c|c|c|}
\hline \multirow{2}{*}{$\begin{array}{c}\text { Features } \\
\text { Datasets }\end{array}$} & \multicolumn{2}{|c|}{ LBP-based Features } & \multicolumn{3}{|c|}{ ULBP-based Features } \\
\cline { 2 - 7 } Results & $\begin{array}{c}\text { Bangla } \\
\text { Part }\end{array}$ & $\begin{array}{c}\text { Hindi } \\
\text { Part }\end{array}$ & $\begin{array}{c}\text { BHSig260 } \\
\text { Dataset }\end{array}$ & $\begin{array}{c}\text { Bangla } \\
\text { Part }\end{array}$ & $\begin{array}{c}\text { Hindi } \\
\text { Part }\end{array}$ & $\begin{array}{c}\text { BHSig260 } \\
\text { Dataset }\end{array}$ \\
\hline EER (\%) & 34.12 & 24.47 & 32.72 & 33.82 & 24.47 & 32.72 \\
\hline Accuracy (\%) & 65.88 & 75.53 & 67.28 & 66.18 & 75.53 & 67.28 \\
\hline
\end{tabular}

From the results obtained concerning the LBP and ULBP features, it is noted that the LBP and ULBP features were almost equally performed in the verification of the BHSig260 dataset and Hindi signatures verification. However, a better result was obtained using the ULBP compared to the LBP features for the Bangla part of the BHSig260 dataset. It is worth mentioning the results obtained using 12 genuine signatures from each signature class for training provided better signature verification results compared to the results obtained considering 8 signatures from each class for training.

The results using the GPDS-100 signature dataset for experiment were also obtained considering the LBP and ULBP features, different values of $K$ and different number of signature samples for training. A summary of the results is presented in Table IX. A graphical representation of the FAR and AER results obtained considering the LBP features and different acceptance/rejection thresholds $\left(\Theta_{c}\right)$ in relation to different values of $\mathrm{K}$ when 8 signatures from the GPDS-100 signature dataset were used for training is further shown in Fig. 3. From Table IX, one can note that the LBP provides better results compared to the ULBP features. This is because, the signatures in the GPDS-100 dataset are small in size and the ULBP provides comparably a compact size feature set than the LBP features. 


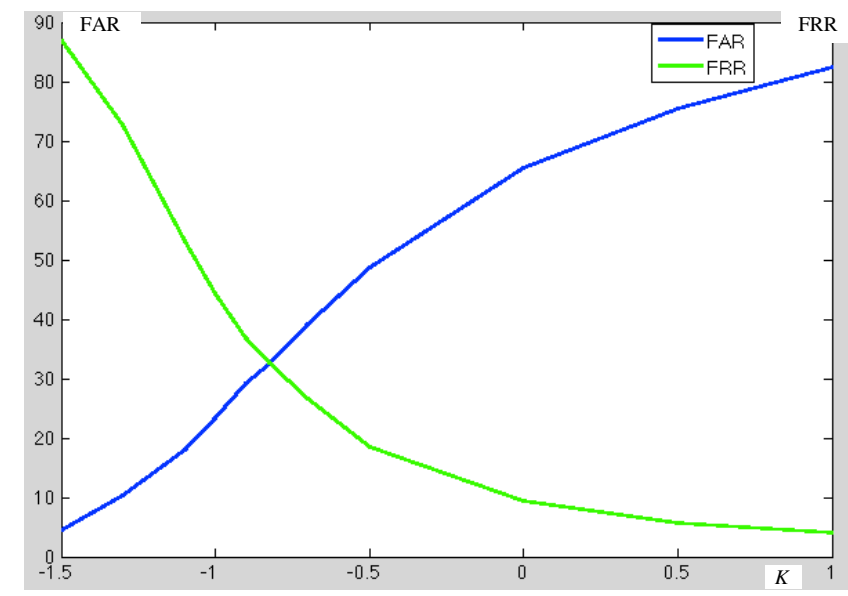

Fig. 3. The graphical representation of the FAR and AER results obtained considering different acceptance/rejection thresholds $\left(\Theta_{c}\right.$ in relation to different values of $K$ when 8 signatures were used for training.

TABLE IX. COMPARISON OF THE RESULTS OBTAINED USING DIFFERENT FEATURES CONSIDERING THE GPDS-100 SIGNATURE DATASET.

\begin{tabular}{|c|c|c|c|c|}
\hline Features & \multicolumn{2}{|c|}{ LBP-based Features } & \multicolumn{2}{|c|}{ ULBP-based Features } \\
\hline Results & $\begin{array}{l}8 \text { signatures } \\
\text { for training }\end{array}$ & $\begin{array}{l}12 \text { signatures } \\
\text { for training }\end{array}$ & $\begin{array}{l}8 \text { signatures } \\
\text { for training }\end{array}$ & $\begin{array}{l}12 \text { signatures } \\
\text { for training }\end{array}$ \\
\hline EER (\%) & 32.31 & 33.06 & 33.38 & 34.31 \\
\hline Accuracy (\%) & 67.46 & 66.94 & 66.62 & 65.69 \\
\hline
\end{tabular}

\section{CONCLUSION}

This study demonstrates an investigation of a signature verification approach involving a large Bangla and Hindi offline signature (BHSig260) dataset introduced in this paper. Texture features based on the LBP and ULBP approaches for off-line signature verification provide reasonably good signature verification results on an Indic-based signature dataset. The BHSig260 dataset and the preliminary results obtained based on the state-of-the-art texture feature extraction methods and the verification approach proposed in this research work can be considered as a benchmark for further investigation on Indic script based signature verification.

\section{REFERENCES}

[1] S. Emerich, E. Lupu, C. Rusu, "On-line signature recognition approach based on wavelets and support vector machines," in Proceedings of the International Conference on Automation Quality and Testing Robotics, pp.1-4, 2010.

[2] M. Kalera, S. Srihari, A. Xu, "Offline signature verification and identification using distance statistics," International Journal of Pattern Recognition and Artificial Intelligence, pp.1339-1360, 2004.

[3] W. Hou, X. Ye, K. Wang, "A survey of off-line signature verification," in Proceedings of the International Conference on Intelligent Mechatronics and Automation, pp. 536-541, 2004.

[4] M. A. Ferrer, F. Vargas, C. M. Travieso, J. B. Alonso, "Signature verification using local directional pattern (LDP)", in Proceedings of the IEEE International Carnahan Conference on Security Technology (ICCST), pp. 336 - 340, 2010.

[5] M. A. Ferrer, J. F. Vargas, A. Morales, A. Ordóñez, "Robustness of offline signature verification based on gray level features”, IEEE
Transaction on Information Forensics and Security, pp. 966-977, 7(3), 2012.

[6] Y. Serdouk, H. Nemmour and Y. Chibani, "Combination of OC-LBP and longest run features for off-line signature verification," in Proceedings of the International Conference on Signal-Image Technology and Internet-Based Systems, pp. 84-88, 2014.

[7] P. Ramesh, P. Nageswara Rao, "Handwritten signature verification based on the use of gray level values," International Journal of Image Processing and Vision Sciences (IJIPVS), pp.50-56, 2(1), 2013.

[6] M. I. Malik, M. Liwicki, and A. Dengel, "Evaluation of local and global features for offline signature verification", in Proceedings of the International Workshop on Automated Forensic Handwriting Analysis, pp. 26-30, 2011.

[7] M.B. Yilmaz, B. Yanikoglu, C. Tirkaz, A. Kholmatov, "Offline signature verification using classifier combination of HOG and LBP features", in Proceedings of the International Joint Conference on Biometrics (IJCB), pp. 1-7, 2011.

[8] J. Ruiz-Del-Solar, C. Devia, P. Loncomilla, F. Concha, "Offline signature verification using local interest points and descriptors", in Proceedings of the 13th Iberoamerican congress on Pattern Recognition: Progress in Pattern Recognition, Image Analysis and Applications, pp. 22-29, 2008.

[9] M. I. Malik, M. Liwicki, L. Alewijnse, W. Ohyama, M. Blumenstein, B. Found, "ICDAR 2013 competitions on signature verification and writer identification for on- and offline skilled forgeries (SigWiComp 2013)," in Proceedings of the ICDAR, pp. 1477-1483, 2013.

[10]S. Pal, U. Pal, M. Blumenstein, "Multi-script off-line signature verification, A two stage approach”, in Proceedings of the International Workshop on Automated Forensic Handwriting Analysis, pp. 31-35, 2013.

[11]S. Pal, U. Pal, M. Blumenstein, "A two-stage approach for English and Hindi off-line signature verification", in Proceedings of the International workshop on Emerging Aspects in Handwritten Signature Processing, pp. 140-148, 2013.

[12]S. Pal, A. Alaei, U. Pal, M. Blumenstein, "Off-line signature verification based on foreground and background information", in Proceedings of the International Conference on Digital Image Computing: Techniques and Applications, pp. 672-677, 2011.

[13]S. Pal, A. Alaei, U. Pal, M. Blumenstein, "Multi-script off-line signature identification", in Proceedings of the International Conference on Hybrid Intelligent Systems, pp. 236-240, 2012.

[14] S. Pal, M. Blumenstein, U. Pal, "Hindi off-line signature verification", in Proceedings of the International Conference on Frontiers Handwriting Recognition, pp. 371-376, 2012.

[15]S. Pal, V. Nguyen, M. Blumenstein, U. Pal, “Off-line bangla signature verification", in Proceedings of the IEEE International Workshop on Document Analysis Systems, pp. 282-286, 2012.

[16] S. Chen, S. Srihari, "Use of exterior contour and shape features inoffline signature verification”, in Proceedings of the ICDAR, pp. 12801284, 2005.

[17] T. Ojala, M. Pietikainen, D. Harwood. "Performance evaluation of texture measures with classification based on kullback discrimination of distributions,” pp. 582-585, 1994.

[18] T. Ojala, M. Pietikainen, T. Maenpaa, "Multiresolution Gray-Scale and Rotation Invariant Texture Classification with Local Binary Patterns,” IEEE Transactions on Pattern Analysis and Machine Intelligence, 24(7), pp. 971-987, 2002.

[19] J. F. Vargas, M. A. Ferrer, C.M. Travieso, J. B. Alonso, "Off-line Handwritten Signature GPDS-960 Corpus," in Proceedings of the of the ICDAR, pp. 764-768, 2007.

[20] J. Ortega-Garcia, J. Fierrez-Aguilar, D. Simon, J. Gonzalez, M. Faundez-Zanuy, V. Espinosa, A. Satue, I. Hernaez, J. J. Igarza, C. Vivaracho, D. Escudero, Q. I. Moro, "MCYT baseline corpus: a bimodal biometric database," IEEE Transactions on Vision, Image and Signal, 150(6), pp. 395-401, 2003. 